\title{
Neonatal Implications of Substance Use Treatment During Pregnancy
}

\author{
Michele K. Savin, MSN, NNP-BC \\ School of Nursing, Thomas Jefferson University
}

Follow this and additional works at: https://jdc.jefferson.edu/nursfp

Part of the Maternal, Child Health and Neonatal Nursing Commons Let us know how access to this document benefits you

\section{Recommended Citation}

Savin, MSN, NNP-BC, Michele K., "Neonatal Implications of Substance Use Treatment During

Pregnancy" (2017). College of Nursing Faculty Papers \& Presentations. Paper 86.

https://jdc.jefferson.edu/nursfp/86

This Article is brought to you for free and open access by the Jefferson Digital Commons. The Jefferson Digital Commons is a service of Thomas Jefferson University's Center for Teaching and Learning (CTL). The Commons is a showcase for Jefferson books and journals, peer-reviewed scholarly publications, unique historical collections from the University archives, and teaching tools. The Jefferson Digital Commons allows researchers and interested readers anywhere in the world to learn about and keep up to date with Jefferson scholarship. This article has been accepted for inclusion in College of Nursing Faculty Papers \& Presentations by an authorized administrator of the Jefferson Digital Commons. For more information, please contact: JeffersonDigitalCommons@jefferson.edu. 


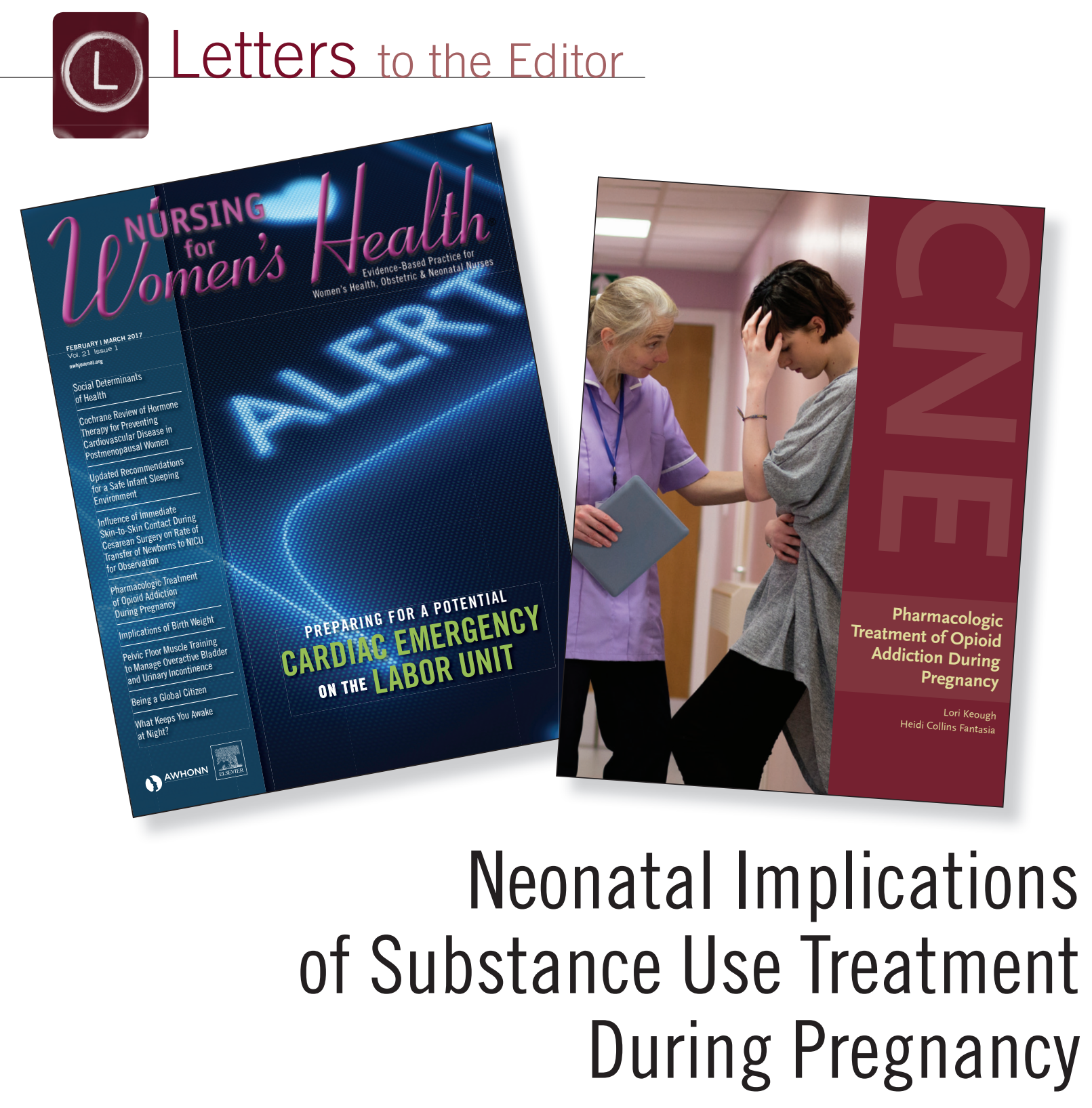

am writing in response to "Pharmacologic Treatment of Opioid Addiction During Pregnancy" by Keough and Fantasia (2017). The article nicely compares and summarizes the use of methadone and buprenorphine to treat substance use in pregnant women. I was dismayed, however, by the sections regarding neonatal considerations and breastfeeding, which contained some problematic assertions and some inaccurate conclusions.

The use of the term addicted to refer to an infant of a woman who is using opioids is inappropriate. The literature clearly differentiates between addiction and dependence; infants are incapable of the psychological activities that define the compulsive nature of addiction. Infants are accurately described in the literature as being born dependent on the substance they have been exposed to. More concerning, however, is the statement that because of the immaturity of all systems, premature infants have more severe withdrawal symptoms. In fact, the exact opposite is true. Although the exact mechanisms for this muted response are not yet delineated in the literature, the Kocherlakota article (2014) cited by the authors is clear in this fact.

Lastly, the paragraph that discusses breastfeeding may be misleading and is not well referenced. Although it is true that breastfeeding is 


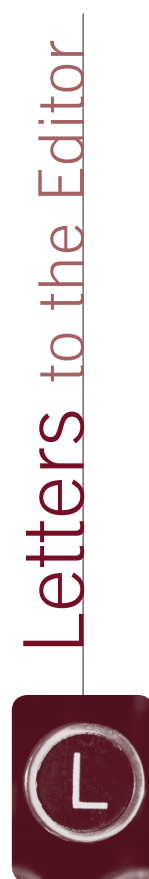

the standard of care, the amount of methadone excreted in breast milk is consistently found to be small and unlikely to cause withdrawal at cessation. The evidence regarding buprenorphine and breastfeeding is limited; however, it also appears that only small quantities of buprenorphine are expressed into breast milk. Guidelines from the Academy of Breastfeeding Medicine (Reece-Stremtan, Marinelli, \& Academy of Breastfeeding Medicine, 2015) clarify the literature on these substances and support breastfeeding as something that will ameliorate symptoms of neonatal abstinence syndrome and may shorten duration of medical treatment required of a neonate. However, the most recent literature does not explain the exact mechanism and points to the act of breastfeeding rather than the breast milk itself as a potential supportive agent. The rate and duration of breastfeeding for substance-exposed mothers are lower than for mothers who are not substance exposed (Wachman, Byun, \& Phillip, 2010; Welle-Strand et al., 2013), making exclusive breastfeeding until introduction of solid food unlikely. There is a strong need for more research in this area.

I appreciate the authors sharing important information on this most challenging of topics, and I hope that these comments may serve to clarify and update the information presented on the neonatal side of the maternal-infant dyad.

\section{Michele Savin, MSN, NNP-BC} Philadelphia, $P A$

(1) CrossMark

\section{References}

Keough, L., \& Fantasia, H. C. (2017). Pharmacologic treatment of opioid addiction during pregnancy. Nursing for Women's Health, 21(1), 34-44. doi:10.1016/j.nwh.2016.12.010

Kocherlakota, P. (2014). Neonatal abstinence syndrome. Pediatrics, 134(2), e547. doi:10.1542/ peds.2013-3524

Reece-Stremtan, S., Marinelli, K. A., \& Academy of Breastfeeding Medicine. (2015). ABM clinical protocol \#21: Guidelines for breastfeeding and substance use or substance use disorder, revised 2015. Breastfeeding Medicine, 10(3), 135-141. doi:10.1089/bfm.2015.9992

Wachman, E. L., Byun, J., \& Philipp, B. L. (2010).
Breastfeeding rates among mothers of infants with neonatal abstinence syndrome. Breastfeeding Medicine, 5(4), 159-164. doi:10.1089/ bfm.2009.0079

Welle-Strand, G. K., Skurtveit, S., Jansson, L. M., Bakstad, B., Bjark, L., \& Ravndal, E. (2013). Breastfeeding reduces the need for withdrawal treatment in opioid-exposed infants. Acta Podiatrica, 102, 1060-1066. doi:10.1111/apa.12378

\section{Authors' Response}

Thanks to Ms. Savin for her thoughtful review of our article. We appreciate the complicated medical and social implications opioid addiction brings to women, particularly those of childbearing age, and to their families. Even more important is the expertise neonatal nurse practitioners can provide to their patients who are vulnerable and unable to care for themselves, as is the case with infants.

The purpose of this article was to present an overview of what is currently known about the pharmacologic treatment of opioid addiction during pregnancy. We applaud Ms. Savin's careful attention to the neonatal component of the maternal-infant dyad. Adding to the complexities of treatment for both women and infants is the often observational and retrospective nature of the research in this area, which carries inherent limits in the development of evidence-based interventions and applicability to clinical practice. The complicated social factors and resulting confounding variables within this population, coupled with significant ethical implications, add to the variability noted in the literature and contribute to the challenges of conducting research in this area.

We are grateful to Ms. Savin for her clinical expertise, perspectives on this important topic, and commitment to the health of women and their infants.

Lori Keough, PhD, MEd, FNP-BC PMHNP-BC Lowell, $M A$

Heidi Collins Fantasia, PhD, RN, WHNP-BC Winthrop, $M A$ 